\section{Avaliação das informações de tuberculose (1989 - 1999) de um Centro de Saúde Escola da cidade de São Paulo}

\section{Evaluation of information on tuberculosis (1989-1999) from a School Health Center in the city of S. Paulo, Brazil}

\author{
Péricles Alves Nogueira \\ Departamento de Epidemiologia \\ Faculdade de Saúde Pública \\ Universidade de São Paulo \\ Avenida Dr. Arnaldo, 715 \\ 01246-904 São Paulo, SP - Brasil \\ pericles@usp.br
}

\section{Maria Ivette Carboni Malucelli \\ Bolsista - Desenvolvimento Tecnológico - Industrial \\ Conselho Nacional de Pesquisa \\ Programa Antártico - brasileiro \\ Pontifícia Universidade Católica do Paraná \\ Avenida Imaculada Conceição, 1155 \\ 80215-030 Curitiba, PR - Brasil}

\author{
Regina Maura Cabral de Melo Abrahão \\ Departamento de Epidemiologia \\ Faculdade de Saúde Pública \\ Universidade de São Paulo \\ Margarida Maria Mattos Brito de Almeida \\ Departamento de Epidemiologia \\ Faculdade de Saúde Pública \\ Universidade de São Paulo
}

\section{Resumo}

As fichas contendo os dados dos pacientes com tuberculose, diagnosticados e/ou tratados em um Centro de Saúde Escola da cidade de São Paulo, eram arquivadas por 10 anos e após esse tempo incineradas. Realizou-se um estudo retrospectivo e descritivo, visando a preservação desta documentação histórica e a avaliação das atividades do Programa de Controle da Tuberculose no período de 1989 a 1999. As informações contidas nas fichas e nos prontuários médicos arquivados foram processadas em uma base de dados, utilizando-se o Programa EPIINFO. Foram analisados o perfil dos pacientes e a evolução do tratamento, para subsídio deste e de outros estudos. Foram tratados 590 pacientes com tuberculose, verificando-se que houve um decréscimo anual no número de pacientes. O maior número dos doentes pertencia ao sexo masculino (58\%), eram solteiros (46,8\%), na faixa etária de 30 a 49 anos $(45,6 \%)$ e residiam nas imediações do Centro de Saúde (48,1\%). A forma pulmonar foi a mais freqüente $(74,4 \%)$ e a baciloscopia foi realizada em 375 pacientes (86,3\%), com uma positividade de $71,7 \%$. O percentual de saída por cura foi de $72,4 \%$, no período estudado, e o de abandono, $12,4 \%$. Concluiu-se que existe a necessidade de melhoria na qualidade das informações, a implantação de um serviço informatizado no atendimento e no seguimento do tratamento, assim como o aprimoramento da busca ativa, ao menos na sua clientela. Deveria também oferecer o tratamento supervisionado, procurando evitar as altas taxas de abandono e transferência observadas neste estudo.

Palavras-chave: Tuberculose. Registros médicos. Centros de Saúde. Estudos de avaliação. 


\section{Abstract}

Files containing data of patients with tuberculosis, diagnosed and/or treated in a School Health Center in the City of S. Paulo, were maintained for 10 years and then were incinerated. A retrospective and descriptive study was conducted, focusing on the preservation of that historical documentation and the evaluation of the activities of the Tuberculosis Control Program during the period from 1989 to 1999. The information contained in the medical archive files was processed into a database utilizing the EPIINFO Program that analyzed the profile of the patients and the results of treatment, so as to support this and other studies. 590 patients with tuberculosis were treated. There was an annual decrease in the number of tuberculosis patients. Most were men (58.0\%), single (46.8\%), between 30 and 49 years of age (45.6\%) and lived in the neighborhood of the Health Center (48.1\%). The pulmonary form was the most frequent (74.4\%) and baciloscopy was performed for 375 patients (86.3\%) with $71.7 \%$ of positive results. $72.4 \%$ of patients were discharged because they were cured and, $12.4 \%$ by default. The conclusion is that the quality of information should be improved; a specific computer program should be implemented for the care of patients and treatment follow-up; case finding should also be improved, at least for the clients of this service. Moreover, treatment should be supervised in order to decrease the high level observed of default.

Keywords: Tuberculosis. Medical records. Health centers. Evaluation studies.

\section{Introdução}

A tuberculose é uma das doenças mais antigas da humanidade e também uma das mais estudadas, entretanto, continua a ser um dos grandes desafios da Saúde Pública em todos os países, principalmente naqueles em desenvolvimento ${ }^{1}$.

Segundo estimativas realizadas pela Organização Mundial da Saúde, um terço da população mundial está infectada pelo Mycobacterium tuberculosis e durante a década de 1990-1999, ocorreriam no mundo 88 milhões de casos novos. Projeções feitas em 1995 indicaram que, no ano 2005, ocorrerão 11,9 milhões de casos novos de tuberculose anualmente ${ }^{2}$.

O Brasil é o décimo país do mundo em número de casos, com cerca de 90.000 casos novos e mais de 5.000 mortes anuais. Destes casos, $48 \%$ se encontram na região sudeste, da qual faz parte o Estado de São Paulo, que por sua vez possui o maior número absoluto de casos do país, apresentando na última década uma incidência de cerca de 17.000 casos novos por ano e um coeficiente oscilando entre 50 e 60 por 100.000 habitantes $^{3,4}$.

O tratamento da tuberculose no Brasil sempre esteve sob a responsabilidade de hospitais e ambulatórios especializados.

Na cidade de São Paulo, um dos ambulatórios que se destacou na luta contra essa endemia foi o Ambulatório de Tuberculose do Centro de Saúde Escola "Geraldo Horácio de Paula Souza”, pertencente à Faculdade de Saúde Pública da Universidade de São Paulo.

A Faculdade de Saúde Pública foi criada em 1918, com o nome de Instituto de Higiene, sob a direção de Samuel Taylor e auxiliado por Geraldo Horácio de Paula Souza.

Paula Souza, em 1922, instalou junto ao Instituto de Higiene um posto de combate à verminose; este foi o embrião do primeiro Centro de Saúde do Brasil, o Centro de Saúde Modelo, anexo ao Instituto de Higiene, destinado ao aprendizado e treinamento do pessoal da Inspetoria de Educação Sanitária e Centros de Saúde (Decreto no 3876 de 11/ 07/25). 
No ano de 1934 foi criado, como uma seção dispensarial, o "Dispensário de Tuberculose" do Instituto de Higiene. Em 1945, por força do decreto-lei 14.857, o Instituto de Higiene passou a ser a Faculdade de Higiene e Saúde Pública e o Centro de Saúde denominado Centro de Aprendizado Urbano, que começou a ter entre seus serviços o Dispensário de Tuberculose. Em 1961, o Centro de Aprendizado Urbano passou a ser denominado Centro de Saúde "Geraldo Horácio de Paula Souza".

Com a integração das atividades de saúde no Estado de São Paulo em 1969, pelo Decreto-lei 50192, o Dispensário de Tuberculose deixou de existir, constituindo-se a Área de Tisiologia do referido Centro de Saúde. Em 1974, foi assinado um convênio entre a Secretaria de Saúde e a Faculdade de Saúde Pública e, a partir desse convênio, o Centro de Saúde dessa Faculdade passou a ser o Centro de Saúde Escola "Geraldo Horácio de Paula Souza” (C.S.E.), vinculado ao Distrito Sanitário do Butantã, da Secretaria de Saúde.

Embora administrativamente subordinada ao C.S.E., a Área de Tisiologia da Faculdade de Saúde Pública da Universidade de São Paulo sempre esteve sob a orientação técnica dos docentes da antiga "Cátedra de Tisiologia" e, atualmente, pelos docentes da "Área de Tisiologia" do Departamento de Epidemiologia da referida Faculdade.

No ano de 1978, foi elaborada uma ficha de controle de tratamento dos doentes de tuberculose do C.S.E. (Anexo 1), cuja finalidade, além do controle, era um registro da evolução do tratamento no decorrer dos anos.

Desde aquele ano esta ficha vem sendo utilizada para todos os doentes, porém, por determinação da Diretoria do C.S.E., os documentos dos pacientes deveriam ficar arquivados por um período máximo de 10 anos e após esse tempo incinerados. Conseqüentemente, foram destruídas as fichas do período de 1978 a 1988.

Visando a preservação desta documentação histórica e com o objetivo de avaliar as atividades do Programa de Controle da Tuberculose desenvolvidas pelo Centro de Saú- de Escola "Geraldo Horácio de Paula Souza”, da Faculdade de Saúde Pública da Universidade de São Paulo, no período de 1989 a 1999, foram analisadas as informações contidas nas fichas dos pacientes com tuberculose, diagnosticados e/ ou tratados neste período.

\section{Métodos}

Realizou-se um estudo retrospectivo e descritivo, com a anuência do Diretor do Centro de Saúde Escola para consulta e coleta dos dados.

Os dados dos pacientes foram obtidos por meio de fichas (anexo 1) e prontuários médicos arquivados no C.S.E. no período $1989-1999$.

As informações contidas nas fichas foram classificadas pela data do diagnóstico (clínico, bacteriológico e/ou radiológico), ordenadas em uma ordem cronológica decrescente.

Após as fichas serem ordenadas e complementadas com informações dos prontuários médicos, programou-se uma base para digitação dos dados, utilizando-se o Programa EPI-INFO.

As variáveis analisadas neste estudo e contidas nas fichas foram: ano do tratamento; idade (nas faixas etárias de 0 a 14 anos, 15 a 29 anos, 30 a 49 anos, 50 a 69 anos e 70 e + anos); estado civil (em casado, divorciado, solteiro, viúvo e ignorado); sexo; localização da tuberculose (em pulmonar, pleural, ganglionar periférica, renal e outras); baciloscopia de escarro; cultura de escarro; exame radiológico de tórax (de acordo com o código da Ficha de Notificação da Tuberculose em: normal, suspeita de TB, outras afecções e não realizado); doenças associadas; esquema de tratamento (de acordo com o Manual de Normas para o Controle da Tuberculose ${ }^{4}$ ); comunicantes; tempo de tratamento (em dias); hospitalização; tipo de saída (em cura, abandono, óbito, transferência e mudança de diagnóstico); tempo de sintomas (em semanas), e local de residência.

O local de residência, para uma melhor análise, foi agrupado nas seguintes regiões: 
Entorno - na qual foram considerados os Distritos vizinhos ao Centro de Saúde Escola (Consolação, Perdizes, Lapa, Pinheiros, Vila Madalena, Jardim América e Cerqueira César); em 5 zonas demográficas do IBGE (Instituto Brasileiro de Geografia e Estatística):Zona Oeste (excluindo-se os Distritos do entorno), Zona Centro (excluindo-se os Distritos do entorno), Zona Sul, Zona Norte e Zona Leste; interior da Grande São Paulo (cidades da região da Grande São Paulo, com exceção da cidade de São Paulo); interior do Estado; outros Estados; sem residência fixa e ignorado.

Após a análise destas variáveis, todos os dados contidos nas fichas foram processados e arquivados em um banco de dados, para posteriores estudos.

As variáveis analisadas foram representadas em tabelas, fornecendo subsídios para a avaliação das atividades de controle da tuberculose no referido Centro de Saúde Escola.

\section{Resultados e Discussão}

No período de $1^{\circ}$ de janeiro de 1989 a 31 de dezembro de 1999, 725 pacientes passaram em consulta e tiveram algum tipo de tratamento no Ambulatório de Tisiologia do Centro de Saúde Escola.

Desses 725 pacientes, foram excluídos 135 deste estudo, dos quais 62 foram tratados por outras doenças respiratórias que não a tuberculose, 29 tinham seqüelas de tuberculose e 44 haviam sido tratados no C.S.E. em anos anteriores a 1989. Os outros 590 eram doentes de tuberculose e iniciaram o tratamento.

Na distribuição desses 590 pacientes, a faixa etária mais atingida pela tuberculose, foi a de 30 a 49 anos (45,6\%), refletindo o que acontece no Brasil e em várias partes do mundo ${ }^{5}$.

Quanto ao estado civil, verificou-se nos solteiros o maior número de pacientes tratados (276 - 46,8\%), provavelmente por estarem mais expostos ao bacilo da tuberculose, devido ao modo de vida mais livre dessas pessoas.
Entre as doenças concomitantes descritas nas fichas, a AIDS foi a mais freqüente. Verificou-se, também, um alto número de casos sem essa informação (492-83,4\%), que poderia refletir tanto os casos de tuberculose sem outra doença concomitante, como a falta de anotação desta ocorrência nas fichas analisadas.

Quanto ao número de hospitalização de pacientes com tuberculose tratados no C.S.E., 35 foram hospitalizados (5,9\%). Havia 263 sem informação $(44,6 \%)$, denotando uma falha no preenchimento das fichas.

Em relação aos esquemas de tratamento, merece destaque o fato de que $93,6 \%$ dos doentes fizeram o esquema I, denotando a preocupação com o cumprimento das Normas de Controle da Tuberculose ${ }^{4,6}$ por parte do C.S.E..

Na Tabela 1 observou-se que a maioria dos doentes pertencia ao sexo masculino, exceto em 1997, quando o número de mulheres doentes foi maior. Além disso, o número de doentes tratados no C.S.E. vem diminuindo ano a ano, sendo que o número mais elevado de casos de tuberculose ocorreu em 1989, com 71 pacientes (12,0\%) e o menor em 1999, com 25 pacientes $(4,2 \%)$.

Entretanto, no Estado de São Paulo, o número de casos notificados de tuberculose em 1989 foi de 14.406 e em 1998 atingiu $18.379^{5}$. No Município de São Paulo, segundo informações da Secretaria da Saúde do Estado, esse número variou de 5.984 em 1989 a 7.708 em 1999, havendo, portanto, um aumento e não uma diminuição do número de casos nestes anos.

O principal motivo da queda do número de doentes no C.S.E. foi a abertura de um Ambulatório de Tisiologia junto ao Departamento de Clínica Médica-Pneumologia do Hospital das Clínicas, em 1996. Com o início das atividades, os pacientes diagnosticados neste hospital, que anteriormente eram encaminhados ao C.S.E., passaram a ser tratados naquele Ambulatório. Além disso, foram criados Centros de Referência para tratamento de AIDS, que também tratavam tuberculose. Outro aspecto importante para a queda no número de pacientes, foi o fato do 
Tabela 1 - Casos de tuberculose segundo sexo e ano de tratamento, Centro de Saúde Escola, São Paulo, 1989 - 1999.

Table 1 - Tuberculosis cases by sex and therapy year, School Health Center in the city of S. Paulo, 19891999.

\begin{tabular}{lcccccc}
\hline \multicolumn{2}{c}{ Ano } & \multicolumn{2}{c}{ Sexo } & \multicolumn{2}{c}{ feminino } & \multicolumn{2}{c}{ Total } \\
& $\mathrm{n}^{\circ}$ & $\%$ & $\mathrm{n}^{\circ}$ & $\%$ & $\mathrm{n}^{\circ}$ & $\%$ \\
\hline 1989 & 45 & 63,4 & 26 & 36,6 & 71 & 100,0 \\
1990 & 34 & 58,6 & 24 & 41,4 & 58 & 100,0 \\
1991 & 43 & 65,2 & 23 & 34,8 & 66 & 100,0 \\
1992 & 35 & 55,6 & 28 & 44,4 & 63 & 100,0 \\
1993 & 35 & 62,5 & 21 & 37,5 & 56 & 100,0 \\
1994 & 32 & 50,0 & 32 & 50,0 & 64 & 100,0 \\
1995 & 36 & 52,9 & 32 & 47,1 & 68 & 100,0 \\
1996 & 26 & 55,3 & 21 & 44,7 & 47 & 100,0 \\
1997 & 15 & 45,5 & 18 & 54,5 & 33 & 100,0 \\
1998 & 25 & 64,1 & 14 & 35,9 & 39 & 100,0 \\
1999 & 16 & 64,0 & 9 & 36,0 & 25 & 100,0 \\
Total & 342 & 58,0 & 248 & 42,0 & 590 & 100,0 \\
\hline
\end{tabular}

C.S.E. adotar uma atitude passiva na busca de casos em sua clientela. Esse decréscimo não pode ser atribuído à falta de médicos, pois desde o final de 1998 o C.S.E. conta com mais um profissional no período da tarde, totalizando dois pneumologistas, havendo a cobertura de um especialista em cada período.

A Tabela 2 demonstrou que o maior número de pacientes com tuberculose (284 -
48,1\%) residiam na região das imediações do Centro de Saúde (região Entorno).

Observou-se também, uma maior concentração de pacientes da zona Sul (60 $10,2 \%)$ os quais, de acordo com os dados contidos nas fichas, eram moradores do bairro de Santo Amaro. Provavelmente, o fato de haver disponibilidade de transporte daquela região para o C.S.E. facilitou o deslocamento dos pacientes, ou ainda, pelo fato

Tabela 2 - Local de residência dos pacientes com tuberculose tratados, Centro de Saúde Escola, São Paulo, 1989 - 1999.

Table 2 - Place of residence of the treated tuberculosis patients, School Health Center in the city of S. Paulo, 1989- 1999.

\begin{tabular}{lrr}
\hline Região & Número & $\%$ \\
\hline Entorno* & 284 & 48,1 \\
Zona Oeste (exceto os distritos do entorno) & 53 & 9,0 \\
Zona Centro (exceto os distritos do entorno) & 25 & 4,3 \\
Zona Sul & 60 & 10,2 \\
Zona Norte & 49 & 8,3 \\
Zona Leste & 43 & 7,3 \\
Interior da Grande São Paulo & 56 & 9,5 \\
Interior do Estado & 2 & 0,3 \\
Outros Estados & 2 & 0,3 \\
Sem residência fixa & 10 & 1,7 \\
Ignorado & 6 & 1,0 \\
\hline Total & 590 & 100,0 \\
\hline
\end{tabular}

*Entorno - região das imediações do Centro de Saúde.

*Around Area - neighborhood area of Health Center 
do C.S.E. ser reconhecido como uma referência para o tratamento da tuberculose.

Nos casos de tuberculose segundo a localização da doença e a faixa etária (Tabela 3), observou-se que houve um maior número de casos de tuberculose pulmonar (439$74,4 \%)$, sendo 276 pacientes $(62,9 \%)$ do sexo masculino e $163(37,1 \%)$ do sexo feminino. A segunda localização mais freqüente foi a pleural, com 56 casos $(9,5 \%)$, sendo 28 $(50,0 \%)$ em cada sexo. A forma ganglionar periférica ocorreu em 36 casos, com 19 pacientes $(52,8 \%)$ pertencentes ao sexo masculino e $17(47,2 \%)$ ao feminino, e a forma renal em $16(2,7 \%)$, sendo $2(12,5 \%)$ em homens e 14 (87,5\%) em mulheres.

Afaixa etária mais freqüente dos casos com tuberculose pulmonar foi a de 30 a 49 anos de idade, com 199 casos (45,3\%), dos quais 142 $(71,4 \%)$ pertenciam ao sexo masculino, e da tuberculose pleural a de 15 a 29 anos, com 25 casos (44,6\%), dos quais 14 (56,0\%) eram homens. Quanto aos casos de tuberculose ganglionar periférica, observou-se que a faixa etária mais freqüente foi a de 30 a 49 anos, com 22 casos $(61,1 \%)$, possivelmente devido à associação TB/HIV, sendo 14 (63,6\%) do sexo masculino. A tuberculose renal atingiu mais a faixa etária de 30 a 49 anos, com 8 casos (50,0\%), sendo $2(25,0 \%)$ do sexo masculino.

Dos 439 pacientes com a forma pulmonar da tuberculose, 375 (85,4\%) realizaram baciloscopia de escarro. Destes 375 pacientes, 269 tiveram baciloscopia positiva $(71,7 \%)$ e 106, negativa $(28,3 \%)$.
Dos 64 pacientes que não realizaram o exame, 11 não conseguiram colher escarro $(17,2 \%)$ e 53 não possuíam essa informação na ficha $(82,8 \%)$.

A cultura de escarro foi realizada em apenas 56 casos (12,8\%) dos 439 com tuberculose pulmonar. Destes 56 pacientes, 35 tiveram resultado positivo $(62,5 \%)$ e 21 negativo (37,5\%). Na maioria dos casos (383 - 87,2\%) a cultura não foi feita, provavelmente porque já chegaram diagnosticados por outros estabelecimentos da área da saúde ou porque somente a partir de novembro de 1996, passou-se a realizar a cultura em todos os materiais biológicos enviados ao Laboratório de Micobactérias. Anteriormente a esta data, a cultura só era realizada nos escarros ou materiais biológicos em cuja requisição médica constasse o pedido deste exame.

Em relação ao exame radiológico de tórax, constatou-se que 411 (93,6\%) dos 439 pacientes com tuberculose pulmonar, possuíam raios-X compatível com a doença.

Dentre os pacientes com tuberculose pulmonar, 336 possuíam a informação a respeito de 487 comunicantes registrados nas fichas e 103 não possuíam essa informação. Dos 487 comunicantes, apenas 216 foram examinados $(44,4 \%)$, denotando uma falha do C.S.E.. Sendo este um Centro de Saúde Escola, deveria haver uma maior preocupação com a descoberta de casos entre os comunicantes.

Entre os 216 comunicantes de pacientes com tuberculose examinados, 57 estavam

Tabela 3 - Casos de tuberculose segundo a faixa etária e a localização anatômica da doença, Centro de Saúde Escola, São Paulo, 1989 - 1999.

Table 3 - Tuberculosis cases by age group and the site of disease, School Health Center in the city of S. Paulo,1989 - 1999.

\begin{tabular}{|c|c|c|c|c|c|c|c|c|c|c|c|c|}
\hline \multirow{2}{*}{$\begin{array}{l}\text { Localização da } \\
\text { tuberculose }\end{array}$} & \multicolumn{2}{|c|}{0 a 14} & \multicolumn{2}{|c|}{15 a 29} & \multicolumn{2}{|c|}{$\begin{array}{c}\text { Faixa etária } \\
30 \text { a } 49\end{array}$} & \multicolumn{2}{|c|}{50 a 69} & \multicolumn{2}{|c|}{$70 e+$} & \multicolumn{2}{|c|}{ Total } \\
\hline & $\mathrm{n}^{\circ}$ & $\%$ & $\mathrm{n}^{\circ}$ & $\%$ & $n^{\circ}$ & $\%$ & $n^{\circ}$ & $\%$ & $n^{\circ}$ & $\%$ & $n^{\circ}$ & $\%$ \\
\hline pulmonar & 3 & 0,7 & 151 & 34.5 & 199 & 45,3 & 70 & 15,9 & 16 & 3,6 & 439 & 100,0 \\
\hline $\begin{array}{c}\text { pleural } \\
\text { ganglionar }\end{array}$ & 1 & 1.8 & 25 & 44,6 & 24 & 42,9 & 4 & 7,1 & 2 & 3,6 & 56 & 100,0 \\
\hline periférica & 1 & 2,8 & 10 & 27,8 & 22 & 61,1 & 1 & 2,8 & 2 & 5,5 & 36 & 100,0 \\
\hline renal & - & - & 3 & 18,7 & 8 & 50,0 & 4 & 25,0 & 1 & 6,3 & 16 & 100,0 \\
\hline outras & 13 & 30,3 & 6 & 13,9 & 16 & 37,2 & 8 & 18,6 & - & - & 43 & 100,0 \\
\hline Total & 18 & 3,0 & 195 & 33,0 & 269 & 45,6 & 87 & 14,8 & 21 & 3,6 & 590 & 100,0 \\
\hline
\end{tabular}


doentes (26,4\%), constatando-se a importância da investigação da doença entre os comunicantes.

Na Tabela 4 observou-se que o percentual de saída por cura foi de $72,4 \%$, no período estudado. Este resultado é inferior ao recomendado pela OMS e adotado pelo Ministério da Saúde, que preconiza uma alta por cura de pelo menos $85,0 \%{ }^{4,7}$. Em apenas 2 anos (1996 e 1998) a meta foi atingida com $85,1 \%$ e $87,2 \%$, respectivamente.

O percentual de saída por abandono foi de $12,4 \%$. As maiores percentagens de abandono foram em 1991 (18,2\%) e 1992 (19,0\%), provavelmente pela falta de medicamentos ocorrida naquele período.

O percentual de óbitos ( $3,7 \%)$ não teve grande variação no período, sendo que nos últimos 3 anos não ocorreram óbitos. $\mathrm{O}$ percentual de transferência $(8,8 \%)$ teve grandes variações no período e o de mudança de diagnóstico foi de $2,7 \%$.

O tempo recomendado de tratamento da tuberculose é de 6 meses $^{4}$. Na Tabela 5 , observou-se que 335 pacientes $(56,8 \%)$ foram tratados por este período de tempo, dos quais 308 tiveram saída por cura (91,9\%), 20 abandonaram o tratamento $(6,0 \%), 4$ mor-

Tabela 4 - Tipo de saída segundo o ano, dos pacientes com tuberculose tratados, Centro de Saúde Escola, São Paulo, $1989-1999$.

Table 4 - Discharge type, by year, of the treated tuberculosis patients, School Health Center in the city of S. Paulo, 1989 - 1999.

\begin{tabular}{|c|c|c|c|c|c|c|c|c|c|c|c|c|}
\hline \multirow{2}{*}{ Ano } & \multicolumn{2}{|c|}{ cura } & \multicolumn{2}{|c|}{ abandono } & \multicolumn{2}{|c|}{$\begin{array}{c}\text { Tipo de saída } \\
\text { óbito }\end{array}$} & \multicolumn{2}{|c|}{ transferência } & \multicolumn{2}{|c|}{$\begin{array}{l}\text { mudança de } \\
\text { diagnóstico }\end{array}$} & \multicolumn{2}{|c|}{ Total } \\
\hline & $\mathrm{n}^{\circ}$ & $\%$ & $n^{\circ}$ & $\%$ & $n^{\circ}$ & $\%$ & $\mathrm{n}^{\circ}$ & $\%$ & $\mathrm{n}^{\circ}$ & $\%$ & $n^{\circ}$ & $\%$ \\
\hline 1989 & 50 & 70,4 & 8 & 11,3 & 2 & 2,8 & 9 & 12,7 & 2 & 2,8 & 71 & 100,0 \\
\hline 1990 & 39 & 67,2 & 9 & 15,5 & 3 & 5,2 & 3 & 5,2 & 4 & 6,9 & 58 & 100,0 \\
\hline 1991 & 47 & 71,2 & 12 & 18,2 & 3 & 4,5 & 3 & 4,5 & 1 & 1,5 & 66 & 100,0 \\
\hline 1992 & 42 & 66,7 & 12 & 19,0 & 4 & 6,3 & 4 & 6,3 & 1 & 1,6 & 63 & 100,0 \\
\hline 1993 & 44 & 78,6 & 8 & 14,3 & 2 & 3,6 & 1 & 1,8 & 1 & 1,8 & 56 & 100,0 \\
\hline 1994 & 42 & 65,6 & 8 & 12,5 & 3 & 4,7 & 7 & 10,9 & 4 & 6,3 & 64 & 100,0 \\
\hline 1995 & 42 & 61,8 & 9 & 13,2 & 3 & 4,4 & 13 & 19,1 & 1 & 1,5 & 68 & 100,0 \\
\hline 1996 & 40 & 85,1 & 1 & 2,1 & 2 & 4,3 & 4 & 8,5 & - & - & 47 & 100,0 \\
\hline 1997 & 27 & 81,8 & 1 & 3,0 & - & - & 4 & 12,1 & 1 & 3,0 & 33 & 100,0 \\
\hline 1998 & 34 & 87,2 & 3 & 7,7 & - & - & 2 & 5,1 & - & - & 39 & 100,0 \\
\hline 1999 & 20 & 80,0 & 2 & 8,0 & - & - & 2 & 8,0 & 1 & 4,0 & 25 & 100,0 \\
\hline Total & 427 & 72,4 & 73 & 12,4 & 22 & 3,7 & 52 & 8,8 & 16 & 2,7 & 590 & 100,0 \\
\hline
\end{tabular}

Tabela 5 - Tempo de tratamento e tipo de saída dos pacientes com tuberculose tratados no Centro de Saúde Escola, São Paulo, 1989 - 1999.

Table 5 - Length of treatment and discharge type of the treated tuberculosis patients, School Health Center in the city of S. Paulo, 1989 - 1999.

\begin{tabular}{|c|c|c|c|c|c|c|c|c|c|c|c|c|}
\hline \multirow{2}{*}{$\begin{array}{l}\text { Tempo de } \\
\text { Tratamento } \\
\text { (dias) }\end{array}$} & \multicolumn{2}{|c|}{ cura } & \multicolumn{2}{|c|}{ abandono } & \multicolumn{2}{|c|}{$\begin{array}{c}\text { Tipo de saída } \\
\text { óbito }\end{array}$} & \multicolumn{2}{|c|}{ transferência } & \multicolumn{2}{|c|}{$\begin{array}{l}\text { mudança de } \\
\text { diagnóstico }\end{array}$} & \multicolumn{2}{|c|}{ Total } \\
\hline & $n^{\circ}$ & $\%$ & $n^{\circ}$ & $\%$ & $n^{\circ}$ & $\%$ & $n^{\circ}$ & $\%$ & $n^{\circ}$ & $\%$ & $n^{\circ}$ & $\%$ \\
\hline$<30$ & - & - & 2 & 11,1 & 4 & 22,2 & 10 & 55,6 & 2 & 11,1 & 18 & 100,0 \\
\hline $31-90$ & - & - & 17 & 37,0 & 5 & 10,9 & 13 & 28,3 & 11 & 23,9 & 46 & 100,0 \\
\hline $91-150$ & - & - & 26 & 53,1 & 7 & 14,3 & 13 & 26,5 & 3 & 6,1 & 49 & 100,0 \\
\hline $151-210$ & 308 & 91,9 & 20 & 6,0 & 4 & 1,2 & 3 & 0,9 & - & - & 335 & 100,0 \\
\hline $211-270$ & 48 & 88,9 & 1 & 1,9 & 1 & 1,9 & 4 & 7,4 & - & - & 54 & 100,0 \\
\hline $271-360$ & 44 & 80,0 & 7 & 12,7 & - & - & 4 & 7,3 & - & - & 55 & 100,0 \\
\hline$>360$ & 27 & 81,8 & - & - & 1 & 3,0 & 5 & 15,2 & - & - & 33 & 100,0 \\
\hline Total & 427 & 72,4 & 73 & 12,4 & 22 & 3,7 & 52 & 8,8 & 16 & 2,7 & 590 & 100,0 \\
\hline
\end{tabular}


reram (1,2\%) e 3 foram transferidos $(0,9 \%)$.

Todas as saídas anteriores a 6 meses de tratamento são por outras causas que não a cura. As saídas com menos de 6 meses totalizaram 113 casos (19,1\%), dos quais 45 abandonaram (39,8\%), 16 morreram $(14,2 \%)$, 36 foram transferidos $(31,8 \%)$ e 16 tiveram mudança no diagnóstico (14,2\%).

Cento e quarenta e dois casos $(24,1 \%)$ ultrapassaram o período de 6 meses de tratamento. Chama a atenção neste grupo que 119 pacientes $(83,8 \%)$ tiveram saída por cura após tratamento prolongado, 8 abandonaram (5,6\%), 2 morreram (1,4\%) e 13 foram transferidos $(9,2 \%)$.

Havia anotação sobre o tempo de sintomas em 280 fichas. Em 79 delas (28,2\%), os pacientes procuraram o serviço em até 4 semanas de sintomas, com tosse e expectoração. Um fato que merece destaque foi o dos que procuraram o C.S.E. com mais de 6 semanas de sintomas (201-71,8\%), evidenciando que um sistema de busca ativa provavelmente teria antecipado o diagnóstico de sintomáticos respiratórios, evitando que estes contagiassem outras pessoas.

\section{Conclusões}

Verificou-se que houve um decréscimo anual no número de pacientes com tuberculose e que existe a necessidade da melhoria na qualidade das informações referentes aos pacientes e seus comunicantes, a implantação de um serviço informatizado no atendimento e no seguimento do tratamento, assim como o aprimoramento da busca ativa, ao menos na sua clientela. Deveria também oferecer o tratamento supervisionado, com a finalidade de evitar as altas taxas de abandono e transferência observadas neste estudo.

\section{Agradecimentos}

Ao Centro de Saúde Escola Geraldo Horácio de Paula Souza da Faculdade de Saúde Pública da Universidade de São Paulo, pela imprescindível colaboração na realização da pesquisa.

À Fundação de Amparo à Pesquisa do Estado de São Paulo (FAPESP), pelo auxílio financeiro-Reserva Técnica-que possibilitou a complementação deste trabalho.

\section{Referências}

1. Hijjar MA. Epidemiologia da tuberculose no Brasil. Inf Epidemiol SUS 1992; 53-87.

2. Raviglione MC, Snider DE, Kochi, A. Global epidemiology of tuberculosis: morbidity and mortality of a worldwide epidemic. JAMA 1995; 273:220-6.

3. Galesi VMN. Mortalidade por tuberculose no Município de São Paulo, análise de uma década, 1986 a 1995 [Dissertação de Mestrado]. São Paulo: Faculdade de Saúde Pública da USP; 1999.

4. Ministério da Saúde. Fundação Nacional de Saúde. Centro Nacional de Epidemiologia. Coordenação Nacional de Pneumologia Sanitária. Manual de normas para o controle da tuberculose. Brasília (DF); 1995.
5. Ministério da Saúde. Fundação Nacional da Saúde. Centro Nacional da Saúde. Centro Nacional de Epidemiologia. Bol Epidemiol 1999. Edição especial.

6. Ministério da Saúde. Secretaria Nacional de Programas Especiais de Saúde. Divisão de Pneumologia Sanitária. Campanha Nacional contra a Tuberculose. Controle da tuberculose: uma proposta de integração ensinoserviço. Brasília (DF); 1994.

7. World Health Organization. WHO Tuberculosis Programme: framework for effective tuberculosis control. Geneva: WHO; 1994. (WHO/TB/94.179). 
ANEXO 1

FACULDADE DE SAÚDE PUBLICA - C S - GERALDO DE PAULA SOUZA

FRENTE AREA DE TISIOLOGIA

NOME

DATA NASCIMENTO

\begin{tabular}{|l|l} 
maDE & COR \\
\hline
\end{tabular}

\begin{tabular}{|c|c|c|}
\hline COR & 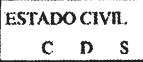 & SEXo \\
\hline
\end{tabular}

ENDERECOO, FONE:

NOME DAMAE

NOME DO PA:

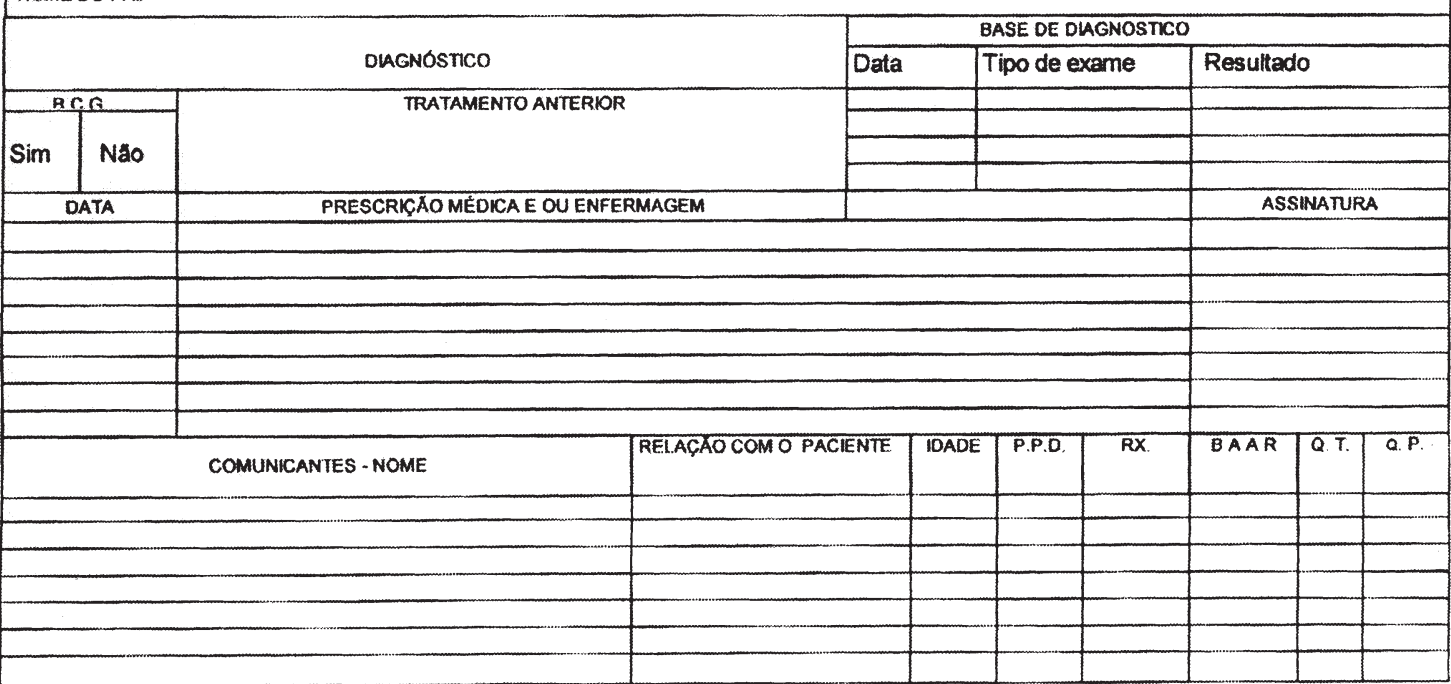

VERSO

MEDICAMENTOS

DATA
EXAME BACTERIOLOGGICO

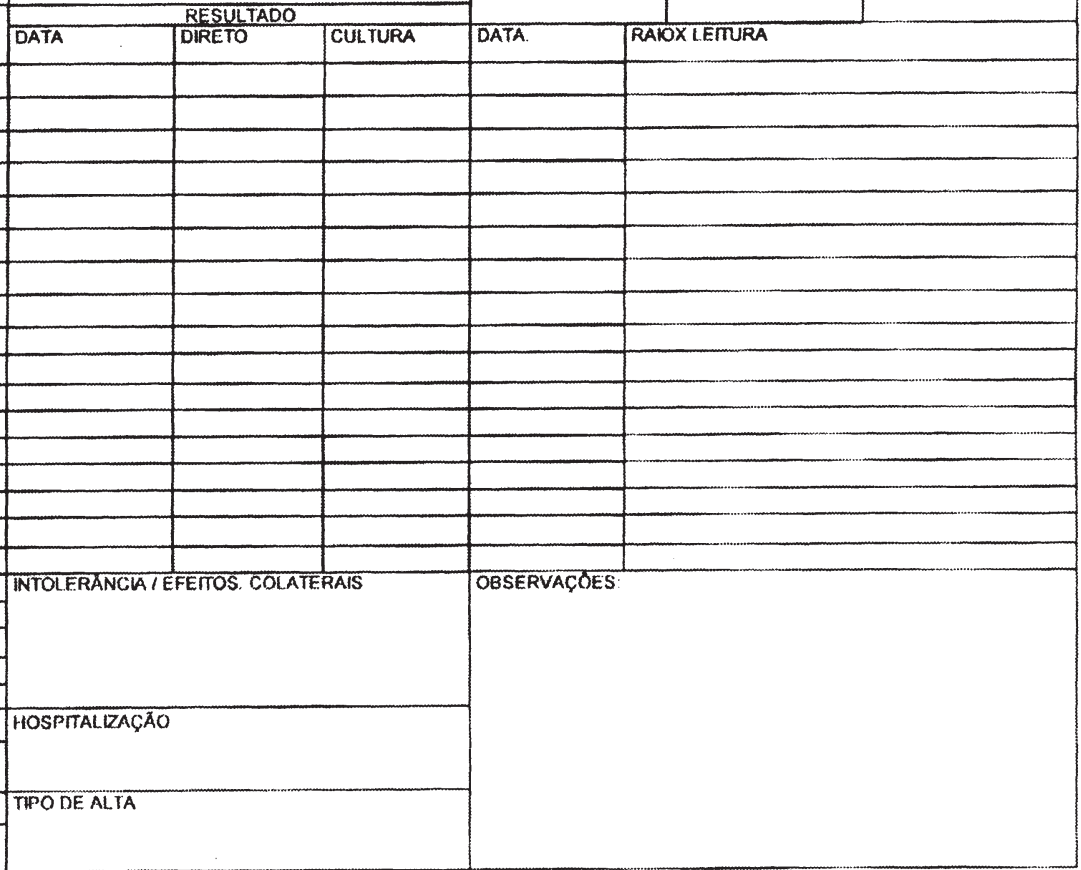

